\title{
Rectangular Rotation of Spherical Harmonic Expansion of Arbitrary High Degree and Order
}

\author{
Toshio Fukushima ${ }^{1,2}$ \\ ${ }^{1}$ National Astronomical Observatory of Japan \\ ${ }^{2}$ Graduate University of Advanced Study SOKENDAI
}

The spherical harmonic expansion is a popular mathematical tool in geodesy and geophysics. This is because its basis functions termed the spherical harmonics satisfy the Laplace equation, and therefore, they are suitable in expanding the geopotential and geomagnetic potential uniformly. Also, the spherical harmonics are proper basis functions to express any kind of analytical function defined on a unit surface such as the topographic height even if it does not satisfy the Laplace equation.

However, the spherical harmonic expansion has a fatal defect: the polar singularity. This is a pure mathematical issue, and therefore, difficult to be resolved. For example, it makes a serious damage in numerically integrating the orbits of artificial satellites passing over the polar regions. Furthermore, it causes a numerical trouble in the computation of the associated Legendre functions of high degree/order, especially near the poles. Refer to Fig. 1. In order to bypass this essential difficulties, we consider moving the polar singularity of arbitrary spherical harmonic expansion to a point on the equator.

For this purpose, by following the approach we adopted in obtaining new type of canonical elements of the orbital and rotational motions (Fukushima, 1994), we recently established a procedure to rotate the expansion around the $y$-axis by 90 degrees such that the $x$-axis becomes a new pole (Fukushima, 2017).

The rotation is conducted by multiplying a matrix composed by special values of Wigner $d$-function (Wigner 1931) with a few normalization constants obtained by simplifying the general results of Aubert (2013). Also, we utilized the symmetry relation of the matrix components (Edmonds, 1957) and the newly discovered decomposi- tion of the matrix multiplication into 6 cases depending on the value and parity of the matrix indices.

Notice that the obtained transformation matrix is unchanged whether the coefficients are $4 \pi$ fully-normalized (Heiskanen and Moritz, 1967) or Schmidt quasinormalized (Winch et al., 2005). Therefore, the developed approach is applicable both to the geopotential usually following the full normalization and the geomagnetic potential typically normalized by the Schmidt quasinormalization.

We compute the matrix components by a set of recurrence formulas. The overflow/underflow problems encountered during the recursive computation of the $d$ functions are effectively resolved by using the so-called X-number formulation (Fukushima, 2012a, 2012b, 2014).

As an example, we obtained the EGM2008R, a set of $2,190 \times 2,190$ coefficients of the rectangular rotated spherical harmonic expansion of the latest geopotential model, the EGM2008 (Pavlis et al., 2012). We confirmed the 18 digit coincidence of the computed geopotential before and after the rotation as seen in Fig. 2.

At any rate, in computing the geopotential accurately, one can switch between the EGM2008 and the EGM2008R depending on the absolute latitude whether being less than 45 degrees or not. Such a proper combination of the original and the rotated expansions will be useful in integrating the polar orbits of artificial satellites precisely, and synthesizing/analyzing the gravitational/geomagnetic potentials and their derivatives accurately in the high latitude regions including the arctic and antarctic area.

The full paper on the present issue was already pub- 
Maximum Error of fnALF in DP X-number Computation

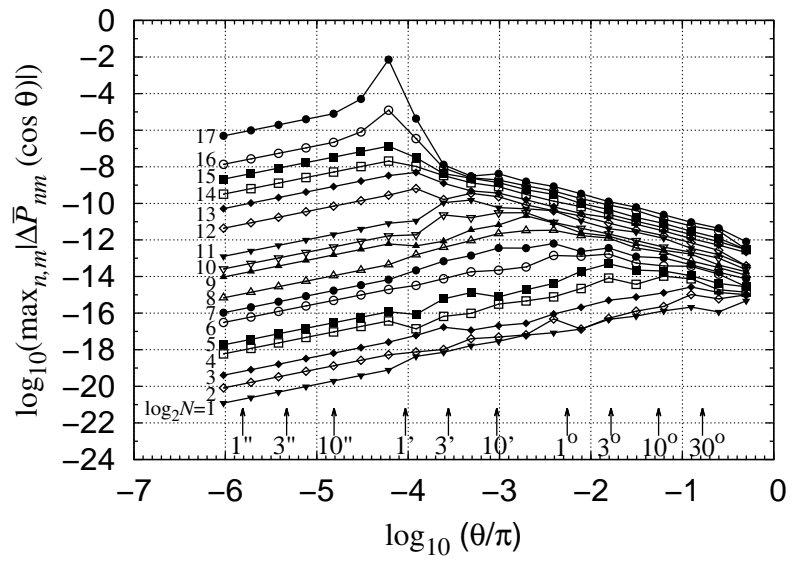

Figure 1: Maximum computation error of the $4 \pi$ fullynormalized associate Legendre functions.

lished (Fukushima, 2017). The PDF file of its reprint and the coefficient file of the EGM2008R are freely available from the following WEB site.

https://www.researchgate.net/profile/Toshio_Fukushima/

\section{References}

Aubert, G., An alternative to Wigner d-matrices for rotating real spherical harmonics. AIP Adv., 3, 062121, 2013.

Edmonds, A. R., Angular Momentum in Quantum Mechanics. Princeton Univ Press, Princeton, 1957.

Fukushima, T., New canonical variables for orbital and rotational motions. Celest. Mech. Dyn. Astron., 60, 5768, 1994.

Fukushima, T., Numerical computation of spherical harmonics of arbitrary degree and order by extending exponent of floating point numbers. J. Geodesy, 86, 271285, 2012a.

Fukushima, T., Numerical computation of spherical harmonics of arbitrary degree and order by extending exponent of floating point numbers: II first-, second-, and third-order derivatives. J. Geodesy, 86, 1019-1028,

\section{$\theta$-Dependence of EGM2008-EGM2008R}

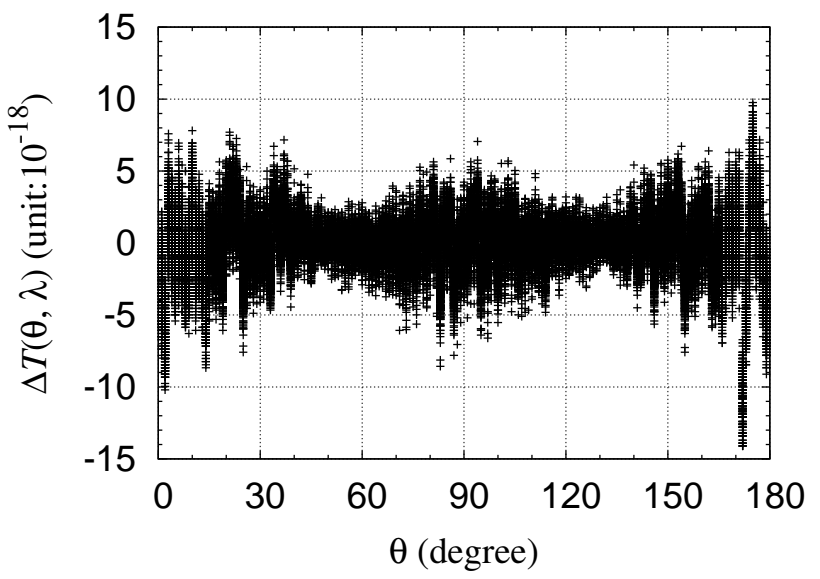

Figure 2: Colatitude dependence of the difference in the geopotential values between EGM2008 and EGM2008R.

$2012 b$.

Fukushima, T., Numerical computation of spherical harmonics of arbitrary degree and order by extending exponent of floating point numbers: III integrals. Comp. Geosci., 63, 17-21, 2014.

Fukushima, T., Rectangular rotation of spherical harmonic expansion of arbitrary high degree and order. J. Geodesy, 91, 995-1011, 2017.

Heiskanen, W. A., and Moritz, H., Physical Geodesy. Freeman, San Francisco, 1967.

Pavlis, N. K., Holmes, S. A., Kenyon, S. C., and Factor, J. K., The development and evaluation of the Earth gravitational model 2008 (EGM2008). J. Geophys. Res., 117, B04406, 2012.

Wigner, E. P., Gruppentheorie und ihre Anwendungen auf die Quantenmechanik der Atomspektren. Vieweg Verlag, Braunschweig, 1931.

Winch, D. E., Ivers, D. J., Turner, J. P. R., and Stening, R. J., Geomangetism and Schmidt quasi-normalization. Geophys. J. Int., 160, 487-504, 2005. 